\title{
Making the invisible visible: the availability and desirability of adherence data in routine CF care- findings from a national
}

\section{questionnaire survey[version 1; peer review: 1 approved, 1}

\section{approved with reservations]}

\author{
Louisa Robinson (1D1, Chin Maguire1, Zhe Hui Hoo (D)2,3, Martin J. Wildman2,3 \\ ${ }^{1}$ Clinical Trials Research Unit, University of Sheffield, Sheffield, South Yorkshire, S1 4DA, UK \\ ${ }^{2}$ Sheffield Adult CF Centre, Sheffield Teaching Hospitals NHS Foundation Trust, Sheffield, Yorkshire, S5 7AU, UK \\ ${ }^{3}$ School of Health and Related Research, University of Sheffield, Sheffield, Yorkshire, S1 4DA, UK
}

\author{
V1 First published: 11 Nov 2019, 8:1904 \\ https://doi.org/10.12688/f1000research.21033.1 \\ Latest published: 17 Jan 2020, 8:1904 \\ https://doi.org/10.12688/f1000research.21033.2
}

\section{Abstract}

Background: Inhaled medications for cystic fibrosis (CF) are effective but adherence is low. Clinicians find it difficult to estimate how much treatment people with CF (PWCF) take, whilst objective adherence measurement demonstrates that patients are poorly calibrated with a tendency to over-estimate actual adherence. The diagnostic approach to a PWCF with deteriorating clinical status and very low adherence is likely to be different to the approach to a deteriorating patient with optimal adherence. Access to objective adherence data in routine consultations could help to overcome diagnostic challenges for clinicians and people with CF. Attitudes of clinicians to the use and importance of routinely available adherence data is unknown. Methods: We conducted an online questionnaire survey with UK CF centres. We asked five questions relating to the current use and perception of objective measurements of adherence in routine care. Results: A total of eight CF centres completed the questionnaire. Few of the responding centres have adherence data readily available in routine clinics ( $13 \%$ of centres use medicines possession ratio; of centres with access to I-nebs $₫$ it was estimated that $17 \%$ of patients had I-neb data regularly available in clinics). All centres considered the availability of objectively measured adherence data to be important. Respondents identified that systems developed to provide adherence data in clinical practice must provide data to both clinicians and patients that is readily understood and easy to use.

Conclusions: Centres perceived the availability of adherence data in routine care to be important but objective measures of adherence is rarely available at present.

\section{Open Peer Review}

Approval Status

1

2

version 2

(revision)

17 Jan 2020

version 1

11 Nov 2019

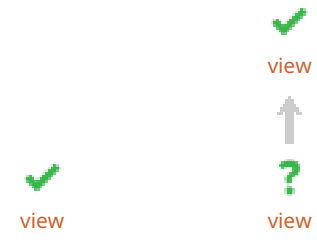

1. Dominique Pougheon Bertrand (iD),

University of Paris 13, Bobigny, France

2. Mark Hew, Alfred Health, Melbourne,

Australia

Monash University, Monash, Australia

Any reports and responses or comments on the article can be found at the end of the article. 


\section{Keywords}

cystic fibrosis, medication adherence, routine monitoring, nebulisers

\section{Corresponding author: Martin J. Wildman (martin.wildman@sth.nhs.uk)}

Author roles: Robinson L: Formal Analysis, Writing - Original Draft Preparation, Writing - Review \& Editing; Maguire C: Conceptualization, Investigation, Methodology, Writing - Review \& Editing; Hoo ZH: Conceptualization, Investigation, Methodology, Writing - Review \& Editing; Wildman MJ: Conceptualization, Methodology, Supervision, Writing - Review \& Editing

Competing interests: Martin Wildman is the Chief Investigator on the CFHealthHub programme that has developed a system to capture and provide adherence data in the UK.

Grant information: This report presents independent research funded by NHS England Commissioning for Quality and Innovation. The views and opinions expressed by authors in this publication are those of the authors and do not necessarily reflect those of the NHS or the Department of Health and Social Care.

The funders had no role in study design, data collection and analysis, decision to publish, or preparation of the manuscript.

Copyright: $\odot 2019$ Robinson L et al. This is an open access article distributed under the terms of the Creative Commons Attribution License, which permits unrestricted use, distribution, and reproduction in any medium, provided the original work is properly cited.

How to cite this article: Robinson L, Maguire C, Hoo ZH and Wildman MJ. Making the invisible visible: the availability and desirability of adherence data in routine CF care- findings from a national questionnaire survey [version 1; peer review: 1 approved, 1 approved with reservations] F1000Research 2019, 8:1904 https://doi.org/10.12688/f1000research.21033.1

First published: 11 Nov 2019, 8:1904 https://doi.org/10.12688/f1000research.21033.1 


\section{Introduction}

Cystic Fibrosis (CF) is a multi-system life-limiting genetic condition in which the most common cause of death is respiratory failure. Daily use of inhaled mucolytic and antibiotic medications are effective to prevent pulmonary exacerbations and preserve lung function ${ }^{1,2}$. Low adherence to $\mathrm{CF}$ medications is associated with poorer health outcomes ${ }^{3}$, yet real-world adherence to inhaled medications is only $35-50 \%$,

Another problem is the potential for diagnostic uncertainties - in a study where the objectively measured adherence was $36 \%$, people with CF reported median adherence of $80 \%$ whilst clinicians estimated adherence rate of $55-60 \%{ }^{5}$. This disparity makes it challenging for healthcare professionals to make informed clinical decisions. The 'invisibility' of adherence in routine care can result in consultations characterised by the 'lamp post syndrome', whereby there is a tendency for clinicians to use readily available information which can be misleading. In other words, clinicians ended up seeking information "where the light is" rather than to use all relevant data sources. Since neither patient self-report nor clinician estimation provides an accurate indication of medication adherence, adherence will be invisible in those centres without systems that make objectively measured adherence visible. This lack of adherence data is critical for diagnosis and assessment. Indeed, the approach in a patient with deteriorating clinical status and low adherence (where treatment failure is due to non-use of existing treatments) is very different to the approach in a patient with deteriorating clinical status and high adherence (where existing treatments have failed, and treatment escalation must be considered).

Furthermore, enabling self-monitoring using objective feedback is associated with increases in target health behaviours ${ }^{7}$ and has been identified as a facilitator in nebuliser adherence ${ }^{8}$. Access to, and feedback from, accurate adherence data could facilitate more effective self-monitoring and self-care for people with $\mathrm{CF}$.

Despite evidence pointing to the importance of objectively measured adherence data in routine $\mathrm{CF}$ care, it remains unclear how frequently clinicians readily have access to objective adherence data in their day-to-day practice and whether such access is desired by clinicians. We therefore conducted an online survey among UK CF centres to establish the perceived importance of routinely available adherence data, and the extent to which this data is currently used in routine care.

\section{Methods}

Centre directors from all 29 UK adult CF centres were invited by email on 12 March 2019 to participate in a short online questionnaire about their views and practices in using adherence data in their day-to-day management of people with CF. Where available, contact details were sought through the CF registry; in the absence of this, email addresses were ascertained through hospital websites.

Centre directors were encouraged to discuss the questions with their clinical colleagues before responding. Centres that did not respond after two weeks were sent two reminders, at two week intervals. All responses were collected within two weeks of the final reminder.

The participant information sheet detailed that responses to the survey would be used for research purposes. The information sheet was embedded within the invitation email. Consent to participate was implied through completion of the questionnaire. The questionnaire was designed with Qualtrics@ 2019 software (version March 2019, Provo, Utah) and consisted of fiveitems with a mixture of Likert scale, percentage estimate and free-text responses (see Extended data). Respondent Internet Protocol (IP) addresses were captured and used to check for duplicate responses. No duplicate responses were captured.

Responses to Likert scale questions (Q1, Q4) were coded 1-5 (1= Very important, 5= Not important) and medians and inter-quartile ranges summarised. For responses to questions requesting a percentage estimate $(\mathrm{Q} 2, \mathrm{Q} 3)$, means and standard deviations were calculated. Free-text responses (Q5) were summarised by extracting key themes.

The study received approval from the School of Health and Related Research (ScHARR) Research Ethics Committee, University of Sheffield (ref: 024042). The survey was hosted on Qualtrics (survey tool approved by the University of Sheffield Corporate Information and Computing Services). The University of Sheffield was the data controller and all survey data exported for analysis was stored on an access restricted folder on the University shared file store.

\section{Results}

A total of eight adult UK CF centres (28\%) provided data from sites across England, Northern Ireland and Wales. Summaries by questionnaire item are summarised in Table 1.

An average of $87 \%$ of patients using inhaled therapies were estimated not to have up to date medicines possession ratio or pharmacy refill data available during a typical outpatient clinic. This included four centres that said $100 \%$ of their patients would not have this information available. Across five centres, an average of $83 \%$ of patients were estimated not to have I-neb® data readily available at the point of consultation. It was stated by two sites that I-nebs were not used at their centre and it is important to recognise that at the time the study, the only routinely available devices to collect objective adherence data were Inebs ${ }^{\circledR}$.

In contrast to low availability of objective adherence data, all centres considered having up-to-date, objective adherence data for inhaled therapies at the point of diagnosis to be at least moderately important; $57 \%$ considered this to be important and a further $29 \%$ considered this to be very important. When asked if they thought that a system able to automatically collect and provide objective, up-to-date adherence data within a consultation would be important, all responding centres said that this would be important to have in their centre and $50 \%$ of respondents felt this would be very important.

A total of seven free-text responses were received for question five regarding the key features for a system to routinely 
Table 1. Questionnaire responses summarised by item (excluding free-text responses to question 5 ).

\begin{tabular}{|c|c|c|c|}
\hline Question & $\mathbf{n}$ & Median (IQR) & n responses $(\%)$ \\
\hline $\begin{array}{l}\text { Q1: A clinical vignette to ascertain whether clinicians desire objective adherence } \\
\text { data at the point of consultation }\end{array}$ & 7 & $2(1-2)$ & $\begin{array}{l}\text { Very important: } 2 \text { (29\%) } \\
\text { Important: } 4 \text { (57\%) } \\
\text { Moderately important: } 1 \text { (14\%) }\end{array}$ \\
\hline $\begin{array}{l}\text { Q4: The perceived importance of a system which provides objective adherence } \\
\text { data at the point of consultation } \\
\text { How important is it to have such a system in your centre? }\end{array}$ & 6 & $1.5(1-2)$ & $\begin{array}{l}\text { Very important: } 3(50 \%) \\
\text { Important: } 3(50 \%)\end{array}$ \\
\hline \multicolumn{4}{|c|}{$1=$ "Very important", 2= "Important", 3= "Moderately important", 4= "Slightly important", 5= "Not important" } \\
\hline & $\mathbf{n}$ & Mean (SD) & \\
\hline $\begin{array}{l}\text { Q2: Proportion of adults whereby pharmacy refill data is readily available at the } \\
\text { point of consultation }\end{array}$ & 6 & $13 \%(31 \%)$ & \\
\hline $\begin{array}{l}\text { Q3: Proportion of adults whereby I-neb® data is readily available at the point of } \\
\text { consultation }\end{array}$ & 5 & $17 \%(21 \%)$ & \\
\hline
\end{tabular}

collect objective adherence data. Multiple respondents stated that "ease of use" would be important, as well as quick and reliable data access. Centres also stated that it would be important for data to be available for both patients and staff to facilitate discussions on adherence.

\section{Conclusion}

Adherence is important for accurate diagnosis, treatment planning and self-management in people with $\mathrm{CF}^{3,6,8}$. We have demonstrated that whilst centres providing care to people with $\mathrm{CF}$ perceive objective adherence data to be important, comprehensive provision of these data is not a feature of current $\mathrm{CF}$ clinical practice. A small percentage of people with $\mathrm{CF}$ have objective measures of adherence available for consultations (between 13-17\% depending on the use of medication possession ratio or I-neb®). This is despite an overall consensus that access to objective adherence data is important for making accurate diagnoses. If a system could produce up-to-date, routinely collected objective adherence data for use in consultations, all centres felt this would be an important asset.

The results of this study demonstrate that in the responding centres clinicians felt that objectively measured adherence at the point of consultation would be useful but was rarely available. A limitation of the current study is that with only 8 out of 29 UK adult centres responding, it is uncertain whether objective adherence data is considered important across the whole of the UK. Nevertheless, it is notable that within the centres that did respond, there was a consensus that objective adherence data was important and no centres that considered objective adherence data to be unimportant.

\section{Ethical approval}

Ethical approval for the collection and analysis of data was obtained from School of Health and Related Research (ScHARR) Research Ethics Committee, University of Sheffield (reference: 024042)

\section{Data availability}

Underlying data

ORDA: Findings from a national questionnaire survey of UK CF centres on availability and desirability of routinely available adherence data, https://doi.org/10.15131/shef.data.10059251.v19.

This project contains the following underlying data:

- CSV file exhibiting the responses to the questionnaire for the eight centres

\section{Extended data}

ORDA: Findings from a national questionnaire survey of UK CF centres on availability and desirability of routinely available adherence data, https://doi.org/10.15131/shef.data.10059251.v19.

This project contains the following extended data:

- Questionnaire sent to the CF centers

Data are available under the terms of the Creative Commons Zero "No rights reserved" data waiver (CC0 1.0 Public domain dedication).
1. Ryan G, Mukhopadhyay S, Singh M: Nebulised anti-pseudomonal antibiotics for cystic fibrosis. In: Ryan G, editor. Cochrane Database Syst Rev. Chichester, UK: John Wiley \& Sons, Ltd. 2003; (3): CD001021. PubMed Abstract | Publisher Full Text

2. Ryan G, Singh M, Dwan K: Inhaled antibiotics for long-term therapy in cystic fibrosis. Ryan G, editor. Cochrane Database Syst Rev. Chichester, UK: John Wiley \& Sons, Ltd. 2011; (3): CD001021.

PubMed Abstract | Publisher Full Text

3. Eakin MN, Bilderback A, Boyle MP, et al.: Longitudinal association between medication adherence and lung health in people with cystic fibrosis. J Cyst 
Fibros. 2011; 10(4): 258-64.

PubMed Abstract | Publisher Full Text | Free Full Text

4. Quittner AL, Zhang J, Marynchenko M, et al:: Pulmonary medication adherence and health-care use in cystic fibrosis. Chest. 2014; 146(1): 142-51.

PubMed Abstract | Publisher Full Text

5. Daniels T, Goodacre L, Sutton C, et al:: Accurate assessment of adherence: selfreport and clinician report vs electronic monitoring of nebulizers. Chest. 2011; 140(2): 425-32.

PubMed Abstract | Publisher Full Text

6. Wildman MJ, Hoo ZH: Moving cystic fibrosis care from rescue to prevention by mbedding adherence measurement in routine care. Paediatr Respir Rev. W.B.

Saunders. 2014; 15 Suppl 1: 16-8.

PubMed Abstract | Publisher Full Text
7. Burke LE, Wang J, Sevick MA: Self-monitoring in weight loss: a systematic review of the literature. J Am Diet Assoc. Elsevier. 2011; 111(1)

$92-102$.

PubMed Abstract | Publisher Full Text | Free Full Text

8. Arden MA, Drabble S, O'Cathain A, et al:: Adherence to medication in adults with Cystic Fibrosis: An investigation using objective adherence data and the Theoretical Domains Framework. Br J Health Psychol. John Wiley \& Sons, Ltd. 2019; 24(2): 357-380.

PubMed Abstract | Publisher Full Text | Free Full Text

9. Robinson L, Maguire C, Wildman M, et al:: Findings from a national questionnaire survey of UK CF centres on availability and desirability of routinely available adherence data. figshare. Dataset. 2019.

http://www.doi.org/10.15131/shef.data.10059251.v1 


\title{
Open Peer Review
}

\section{Current Peer Review Status:}

\section{Version 1}

Reviewer Report 03 January 2020

https://doi.org/10.5256/f1000research.23149.r58100

(C) $2020 \mathrm{Hew}$ M. This is an open access peer review report distributed under the terms of the Creative Commons Attribution License, which permits unrestricted use, distribution, and reproduction in any medium, provided the original work is properly cited.

\author{
Mark Hew \\ ${ }^{1}$ Alfred Health, Melbourne, Vic, Australia \\ 2 School of Public Health and Preventive Medicine, Monash University, Monash, Australia
}

This manuscript describes a survey of adherence monitoring availability, and views regarding the benefits of such, in the context of UK national CF centres.

Aims, methods, results are described in sufficient detail for the reader to understand and follow. The discussion is reasonable and does not exceed the scope of the data.

The results demonstrate substantial limitations in clinicians' ability to capture and utilise adherence data in clinical care- findings important to highlight and necessary to address.

I have the following major comments:

1. The $8 / 29$ response rate from centre directors is disappointing. This may therefore represent significant selection bias and this bias should be explicitly mentioned in the limitations; i.e. centres with less interest in an adherence monitoring system are less likely to respond.

2. The sub-optimal response rate may also indicate limited interest or engagement among some centres for implementing a concerted adherence monitoring system. The authors might consider including this in their discussion, in a sensitive and tactful manner.

3. While the respondents agreed a user-friendly system to make adherence data available in consultations was desirable, they were not asked what barriers currently stood in the way of implementing this- (staff, equipment, time). If this survey is repeated in future, this would yield useful information to develop and implement such a system.

4. As a practitioner outside the CF field, I am surprised that adherence monitoring is not a routine part of routine assessment, especially prior to the use of eye-wateringly expensive advanced therapies. For example, the Severe Asthma centres in the UK are tasked with ensuring objective adherence data is acceptable prior to commencing biologics (far less expensive than gene potentiators), and the Australian Pharmaceutical benefits system requests that adherence is addressed prior to prescription of biologics (although objective measurement is not a requirement here). While clinicians are naturally (and, I judge, 
correctly) reluctant to set adherence thresholds requirements prior to implementing advanced therapies, knowledge of adherence patterns is always desirable when contemplating stepping-up any therapy. The authors may consider drawing comparisons between the practice in CF and severe asthma fields even in the UK alone.

Is the work clearly and accurately presented and does it cite the current literature? Yes

Is the study design appropriate and is the work technically sound?

Yes

Are sufficient details of methods and analysis provided to allow replication by others? Yes

If applicable, is the statistical analysis and its interpretation appropriate?

Not applicable

Are all the source data underlying the results available to ensure full reproducibility? No source data required

Are the conclusions drawn adequately supported by the results? Yes

Competing Interests: No competing interests were disclosed.

Reviewer Expertise: Evidence based health care, severe asthma, allergic disease, pleural disease

I confirm that I have read this submission and believe that I have an appropriate level of expertise to confirm that it is of an acceptable scientific standard, however I have significant reservations, as outlined above.

Author Response 17 Jan 2020

Louisa Robinson, University of Sheffield, Sheffield, UK

Dear Professor Hew,

Please see our responses to your comments below and refer to the revised article which has now been published.

1. The $8 / 29$ response rate from centre directors is disappointing. This may therefore represent significant selection bias and this bias should be explicitly mentioned in the limitations; i.e. centres with less interest in an adherence monitoring system are less likely to respond.

We agree this is an important bias and have now included this in the new 3rd paragraph of the conclusion. The sentence now reads: 
"Centres with less interest in monitoring adherence may be less likely to respond, so it is uncertain whether objective adherence data is considered important across the whole of the UK."

2. The sub-optimal response rate may also indicate limited interest or engagement among some centres for implementing a concerted adherence monitoring system. The authors might consider including this in their discussion, in a sensitive and tactful manner.

We agree this is an important point and have now added this in the 1st paragraph of the conclusion. The sentence now reads:

"Only a small percentage of people with CF have objective measures of adherence available for consultations (between 13-17\% depending on the use of medication possession ratio or I-neb ${ }^{\circledR}$ ) with this figure likely to be even lower among centres which did not respond to the survey."

3. While the respondents agreed a user-friendly system to make adherence data available in consultations was desirable, they were not asked what barriers currently stood in the way of implementing this- (staff, equipment, time). If this survey is repeated in future, this would yield useful information to develop and implement such a system.

This is a good suggestion and we have now added the following sentence in the 2 nd paragraph of the conclusion:

"To help embed an objective adherence monitoring system within routine CF clinical practice, it would be useful to ascertain the barriers for implementing such a system among CF centres in future surveys."

4. As a practitioner outside the CF field, I am surprised that adherence monitoring is not a routine part of routine assessment, especially prior to the use of eye-wateringly expensive advanced therapies. For example, the Severe Asthma centres in the UK are tasked with ensuring objective adherence data is acceptable prior to commencing biologics (far less expensive than gene potentiators), and the Australian Pharmaceutical benefits system requests that adherence is addressed prior to prescription of biologics (although objective measurement is not a requirement here). While clinicians are naturally (and, I judge, correctly) reluctant to set adherence thresholds requirements prior to implementing advanced therapies, knowledge of adherence patterns is always desirable when contemplating stepping-up any therapy. The authors may consider drawing comparisons between the practice in CF and severe asthma fields even in the UK alone.

This is a very important point and we have now drawn such a comparison in the 2 nd paragraph of the conclusion:

"The situation in CF contrasts with that of other long-term respiratory conditions such as asthma whereby adherence measurement is mandated prior to commencing expensive biologics e.g. mepolizumab."

Competing Interests: No competing interests were disclosed. 
Reviewer Report 30 December 2019

https://doi.org/10.5256/f1000research.23149.r57625

(C) 2019 Pougheon Bertrand D. This is an open access peer review report distributed under the terms of the Creative Commons Attribution License, which permits unrestricted use, distribution, and reproduction in any medium, provided the original work is properly cited.

\title{
Dominique Pougheon Bertrand
}

\author{
LEPS EA 3412, University of Paris 13, Bobigny, France
}

This article deals with the adherence of adult patients with CF to the nebulization treatments and more particularly the importance of assessing their adherence in clinic consultations to establish a good diagnosis of the patient's health (a deterioration might be due to non-adherence to a prescribed treatment or other causes despite good adherence to the treatments) and thus to offer the best treatment strategies to the patient.

The purpose of the study is to assess the availability and desirability, for the clinicians involved in CF care in the UK, of objective adherence data from their patients. Five questions were asked through an online questionnaire survey to the UK Adult CF centres. Availability refers to Medical Possession Ratio (MPR - refill data) or I-neb data (a device used to nebulize various treatments) available during the clinic visit. Desirability refers to the value that clinicians place on this data at the point of consultation.

Though clinicians place importance on objective adherence data during the clinic visits of the patients, this data is rarely available (only available for about $15 \%$ patients) and their provision not a feature of CF care in current clinical practice. The author indicates as a limitation of the study the fact that the response rate to the survey is only $28 \%$ of the CF centers ( 8 centers out of 29 ).

This paper is very clear, the methods is presented in details so it could be replicated in another study, and the results are fully presented. It gives perspectives to improve both the quality of care and the health outcomes of the patients by increasing the availability and use of patient adherence data to nebulized treatments. This would increase the relevance of prescriptions and possibly open up a discussion with the patient about the feasibility of their treatments and routines necessary to sustain care in daily life.

May the author consider citing the following paper ( $6^{\text {th }}$ position in the reference) as it is an assessment of adherence in CF patients for inhaled therapies in France: Heloïse Rouzé et al. ${ }^{1}$

\section{References}

1. Rouzé H, Viprey M, Allemann S, Dima AL, et al.: Adherence to long-term therapies in cystic fibrosis: a French cross-sectional study linking prescribing, dispensing, and hospitalization data. Patient Prefer Adherence. 2019; 13: 1497-1510 PubMed Abstract | Publisher Full Text

\section{Is the work clearly and accurately presented and does it cite the current literature?} Yes 
Is the study design appropriate and is the work technically sound?

Yes

Are sufficient details of methods and analysis provided to allow replication by others? Yes

If applicable, is the statistical analysis and its interpretation appropriate?

Yes

Are all the source data underlying the results available to ensure full reproducibility? Yes

Are the conclusions drawn adequately supported by the results?

Yes

Competing Interests: No competing interests were disclosed.

Reviewer Expertise: Public Health, Quality Improvement, Patient Experience, Qualitative methodology (grounded theory), Cystic Fibrosis.

I confirm that I have read this submission and believe that I have an appropriate level of expertise to confirm that it is of an acceptable scientific standard.

The benefits of publishing with F1000Research:

- Your article is published within days, with no editorial bias

- You can publish traditional articles, null/negative results, case reports, data notes and more

- The peer review process is transparent and collaborative

- Your article is indexed in PubMed after passing peer review

- Dedicated customer support at every stage

For pre-submission enquiries, contact research@f1000.com 